\title{
Review of: "A living bdelloid rotifer from 24,000-year- old Arctic permafrost"
}

\author{
Aydin Örstan
}

Potential competing interests: The author(s) declared that no potential competing interests exist.

\section{Rotifers from the deep freezer}

I first encountered bdelloid rotifers soon after I had taken up microscopy as a hobby in my early 20s. I was fascinated when I learned that they could survive drying and started doing simple experiments to test their prowess. That was more than 40 years ago, but bdelloids have not ceased to amaze me. In a recent publication, Shmakova et al. (2021) reported yet another feat accomplished by bdelloids: they were recovered alive from permafrost soil removed $3.5 \mathrm{~m}$ below the surface in northeastern Siberia. At that depth the temperature was $-6^{\circ} \mathrm{C}$. The soil sample was carbon-dated to approximately $24,000 \mathrm{BP}$.

That bdelloids can survive freezing is not surprising. One of the most abundant invertebrates in the meltwater ponds in Antarctica is the bdelloid Philodina gregaria that was discovered by the microscopist James Murray during the British Antarctic Expedition of 1907-9. Murray (1910) could obtain large numbers of these rotifers in the winter by thawing ice containing plants from the lakes. Besides $P$. gregaria, there are other species of bdelloids that are endemic to Antarctica (lakovenko et al., 2015). As Shmakova et al. demonstrate, bdelloids from temperate climates also survive freezing. Storage at very low temperatures may, in fact, be the perfect method for the long-term preservation of dry bdelloids. I have been conducting a prolonged experiment to monitor the survival at $-21^{\circ} \mathrm{C}$ of bdelloids obtained from a dry birdbath. In a sample rewarmed and rehydrated after five years, $98 \%$ of the rotifers were alive. Of course, five years is a blink of the eye compared to 24,000 years.

But how can bdelloids, which are aquatic animals that can be active only when immersed in water, survive being frozen and dry for any length of time? In the frozen state the chemical reactions that constitute the metabolism would be extremely slow. If an animal was dry when it was frozen or if any ice that may have been present initially was lost through sublimation, it would be even more difficult to explain the functioning of the metabolism without the participation of water molecules. If the metabolism stops, then life must survive as a purely structural entity. The beginnings of the dichotomous treatment of life as metabolism versus structure is more than 100 years old. In 1878, Claude Bernard was already using a flame as a metaphor for the cellular metabolism: "a living being is like a burning torch: the body wastes, the substance of the torch burns; the first shines with a physical flame, the other with a vital flame." 
However, once the pioneering experiments demonstrated that bacteria and plant seeds could survive temperatures below $-200^{\circ} \mathrm{C}$, an opposing viewpoint appeared: “...life may be completely arrested by cold and again revived by heat-if the molecular structure characteristic of living protoplasm (whatever that may be) remains unchanged" (Le Conte, 1901; italics original).

Obviously, life is both metabolism and structure. In the case of bdelloids and a few other animals that can survive being completely dry or frozen, for example, tardigrades and nematodes, the reversibility of the passage from active to inert structure is what constitutes life and sets it apart from all other chemical or physical phenomena that also consume energy to maintain their organizations. There is no such thing as an extinguished flame; once it is deprived of oxygen or fuel, a flame ceases to exist. In contrast, a bdelloid is able to survive as a dry or frozen inert molecular organization that is ready to start metabolizing once the water returns or the temperature rises.

The permafrost bdelloid is apparently a previously unknown species in the genus Adineta.

Shmakova et al. place the closest genetic relatives of their bdelloid in a group that they refer to as the Adineta vaga complex. This is a sensible approach. The taxonomy of bdelloid rotifers is in need of an overhaul and I have argued elsewhere that Adineta vaga sensu stricto (Davis, 1873) is not a recognizable taxon (Örstan, 2020). There are probably not just one, but several complexes of genetically different and morphologically similar Adineta species waiting to be resolved. I am looking forward to the publication of the formal description of the new species.

Whenever an extraordinary claim is encountered, a scientist must always be skeptical. In this case, the possibility of the revived rotifers' actually being present-day animals that had accidentally been introduced to the permafrost sample should be taken into account. Bdelloid rotifers are ubiquitous in environments that are permanently or periodically wet and may be found in a wide range of habitats, including mosses, recurrent rain puddles, rock pools and wet soil. Shmakova et al. explain both the field and the subsequent laboratory procedures they used to prevent the contamination of the extracted sample from the upper layers of the soil and from other sources. My one concern is that bdelloids were seen in one of the primary cultures only after the passage of at least 20 days. Is this indicative of contamination of that particular culture? Perhaps, but the precautions taken in the laboratory make that unlikely. The authors also searched for the sequences of the revived bdelloids in a metagenome obtained from the same soil sample. Unfortunately, the results of this analysis are not conclusive, but, in the authors' own words, "strongly suggest" that the revived rotifers originated from the permafrost layer.

We should, therefore, retain a sliver of doubt about the validity of the extraordinary claim that bdelloids can remain alive frozen for 24,000 years. Hopefully, future studies will validate this result and the bdelloid rotifers-frozen or not-will continue to amaze us. 


\section{References}

Bernard, C. 1878. The definition of life. Popular Science Monthly, supplement, 7-12:511-524.

lakovenko, N.S., Smykla, J., Convey, P., Kašparová, E., Kozeretska, I.A., Trokhymets, V., Dykyy, V.I., Plewka, M., Devetter, M., Duriš, Z., Janko, K. 2015. Antarctic bdelloid rotifers: diversity, endemism and evolution. Hydrobiologia 761: 5-43.

Le Conte, A. 1901. What is life? Science 13:991-992.

Murray, J. 1910. Antarctic Rotifera. British Antarctic Expedition 1907-9, Reports on the Scientific Investigations 1: 41-65.

Örstan, A. 2020b. The trouble with Adineta vaga (Davis, 1873): a common rotifer that cannot be identified (Rotifera: Bdelloidea: Adinetidae). Zootaxa 4830: 597-600. https://doi.org/10.11646/zootaxa.4830.3.8

Shmakova, L., Malavin, S., lakovenko, N., Vishnivetskaya, T., Shain, D., Plewka, M., Rivkina, E. 2021. A living bdelloid rotifer from 24,000-year-old Arctic permafrost. Current Biology 31:R712-R713.

https://doi.org/10.1016/j.cub.2021.04.077 